\title{
ADAPTIVE COMPLEX WAVELET-BASED FILTERING OF EEG FOR EXTRACTION OF EVOKED POTENTIAL RESPONSES
}

\author{
Arnaud Jacquin \\ Everest Biomedical Instruments \\ arnaudj@everest-co.com
}

\author{
Roy John \\ NYU Medical Center Carnegie Mellon University \\ roy@brl4.med.nyu.edu jelenak@.cmu.edu
}

\begin{abstract}
We propose a new method for the extraction of Auditory Brainstem Responses (ABRs) from an EEG signal. It is based on adaptive filtering of signals in the wavelet domain, where the transform used is a nearly shift-invariant Complex Wavelet Transform (CWT). We compare our algorithm to two existing methods: The first simply consists of bandpass filtering the input EEG signal followed by linear averaging. The second method uses signal-adaptive filtering in the Fourier domain based on phase variance computed at each spectral component of the FFT. Realistic models of EEG and ABR are generated for this comparison. Results show that the wavelet-based method consistently outperforms the other two methods for ABR signals with an initial signal-to-noise ratio less than $-20 \mathrm{~dB}$.
\end{abstract}

\section{INTRODUCTION}

Auditory Evoked Potential (AEP) signals are transient electrical biosignals produced by various regions of the human brain in response to auditory stimuli (such as a periodic repetition of "clicks"). These signals are traditionally categorized into: an Auditory Brainstem Response (ABR) which occurs during the first $11 \mathrm{~ms}$ after the stimulus, followed by a Mid-Latency cortical Response (MLR) which is typically confined to the next $70 \mathrm{~ms}$, followed by a slow cortical response which starts at about $80 \mathrm{~ms}$ after the stimulus.

ABR signals have a waveform morphology which typically exhibits five waves (peaks) in the 1.5 to $6 \mathrm{~ms}$ post-stimulus interval [9]. Specific deviations from a "normal" morphology can be mapped to specific auditory dysfunctions or specific neurological or psychiatric disorders making these signals of great interest for clinical diagnostic purposes.

EP signals are typically one order of magnitude smaller than the EEG signal. Standard algorithms for the extraction of EP signals from the EEG fundamentally rely on bandpass filtering the EEG followed by averaging EEG frames (also called "epochs") synchronously with the beginning of each stimulus. This method implicitly assumes that the EEG is a stationary, zero-mean process and thus, averaging eliminates the average EEG and leaves only the average EP.

We focus here on the extraction of ABR signals. The first description of the human ABR was made by Jewett and Williston [10] in 1971. Works describing techniques for extracting the ABR from the EEG are given in $[1,2,3,4,7,8$, 9]. Here, we poropose a new algorithm of adaptive filtering in the time-frequency domain using a specific Wavelet Transform and compare it to traditional ABR extraction in the form of bandpass filtering followed by averaging as well as adaptive filtering in the Fourier domain [8].

\section{EEG SIGNAL MODELS}

The advantage of developing mathematical models for biosignals is that it allows us to objectively compare the performance of different algorithms. In this work, we used a mathematical model of the EEG (the "noise"), which produces signals at seven electrode locations referred to as Fp1, Fp2, F3, F4, F7, F8, and Fz. Each EEG signal has a power spectrum which is close to that of a real-world EEG, i.e. is proportional to $(1 / f)^{\alpha}$, where $f$ is the frequency in Hz. We assumed a sampling frequency of $10 \mathrm{kHz}$, which is sufficient for ABR extraction since power spectral estimates of ABR signals show little energy at frequencies above $1500 \mathrm{~Hz}$ [9].

For models of ABR responses, we used typical averaged responses taken from [9] over an analysis epoch of either 12 or $15 \mathrm{~ms}$. These models, referred to respectively as "pa04ABR" and "pa07ABR" are shown in Fig. 1, where peaks are labeled by roman numerals as per the convention proposed by Jewett in [10]. As far as our study, these models can be seen as "ideal" (i.e. noise-free) EP responses. A final, "simulated EEG," containing the embedded ideal EP $S[n]$ was obtained by adding the ideal signal to each consecutive epoch of the EEG, thereby producing a noisy signal $E[n]$ :

$$
E[n]=S[n]+N[n]
$$

where $N[n]$ represents the biological noise contributed by the EEG. Note that adding the same ideal signal to each EEG frame assumes that there is no latency jitter in the ABR response.

To measure reconstructed signal quality, we use the signalto-noise ratio (SNR). Suppose that a given signal extraction method produces an estimate $S_{r e c}[n]$. The measure of distortion provided by the $\mathrm{SNR}$, computed in $\mathrm{dB}$, is then:

$$
\operatorname{SNR}\left(S, S_{\text {rec }}\right)=10 \log _{10}\left(\operatorname{var}(S) / \operatorname{var}\left(S-S_{\text {rec }}\right)\right),
$$

where $\operatorname{var}(S)$ indicates the variance (power) of $S$.

\section{TRADITIONAL ABR EXTRACTION}

\subsection{Bandpass Filtering and Synchronized Averaging}

The average input SNR (before any averaging or filtering) for signals "pa04ABR" and "pa07ABR" is $-23.2 \mathrm{~dB}$ and $-26.6 \mathrm{~dB}$ 
respectively. If signal and noise were sinusoidal signals, these values would correspond to ratios of noise amplitude over signal amplitude of 14 and 21 respectively (using (2)). These numbers are typical of actual ABR recordings and demonstrate that ABR responses cannot be visually detected from single sweeps of EEG.

The traditional way to improve the SNR and thereby extract an Evoked Potential signal from the EEG consists of first filtering the EEG using a bandpass filter suitably adapted to the type of EP of interest, followed by averaging a (possibly large) number of epochs all synchronized to the beginning of the stimulus. For ABR signals, different filter passbands have been used, with highpass cutoff frequency in the range $30-300 \mathrm{~Hz}$ and lowpass cutoff between $1500 \mathrm{~Hz}$ and $3000 \mathrm{~Hz}$. For the purpose of this work, we decided to select a fairly wide passband of 30 $3000 \mathrm{~Hz}$, as recommended by Hall in [9].
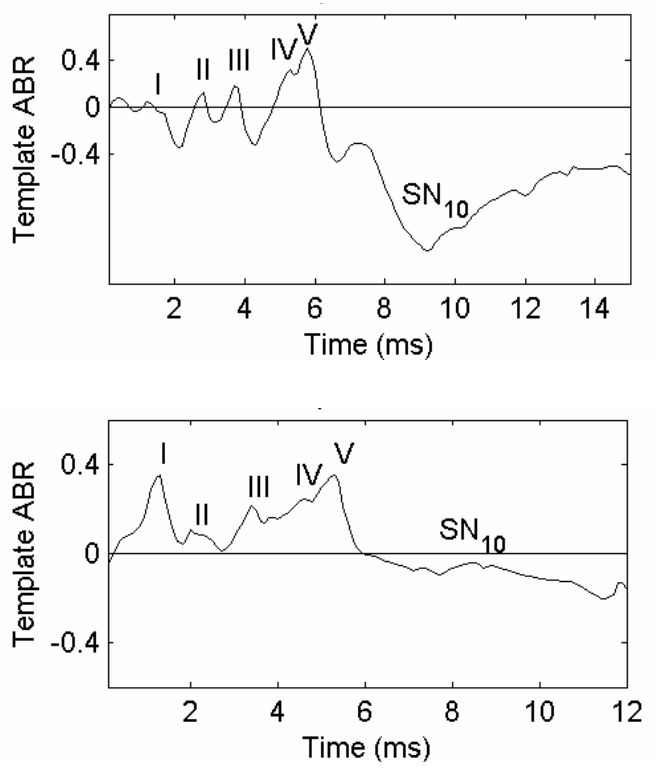

Figure 1: Template noise-free ABR signals "pc04ABR" (top) and "pc07ABR" (bottom). The vertical axis is in microvolts.

\subsection{Optimal Adaptive Filtering in the FFT Domain}

The optimal digital filtering method developed in [8] showed that denoised "light-average" ABR signals could be obtained by processing these averages in the Fourier domain. This algorithm initially segments EEG data into a set of $K$ "trials" or "subaverages" of $M$ frames each. We allowed these trials to overlap by a number of $P$ frames $(P<M)$. For each trial, spectral analysis is performed using an $L$-point ${ }^{1}$ Fast Fourier Transform (FFT). The phase variance across trials of each normalized complex spectral component is computed according to:

$$
\operatorname{Var}\left(\psi_{i}\right)=(1 / K) \sum_{j=1}^{K}\left|h_{i j}-h_{i}\right|^{2},
$$

with

${ }^{1}$ Since $L$ is chosen to be larger than the frame length $N$, we padded the frames by their mean values.

$$
h_{i j}=H_{i j} /\left|H_{i j}\right| \text {, and } h_{i}=(1 / K) \sum_{j=1}^{K} h_{i j} \text {, }
$$

where $H_{i j}$ is the $i^{\text {th }}$ complex spectral component of the $j^{\text {th }}$ trial, $h_{i j}$ is the normalized spectral component and $h_{i}$ is the mean normalized component. A low phase variance for any given spectral component indicates that this component is likely to belong to the phase-locked, repeatable EP signal, whereas a high phase variance is likely to be due to (random) noise. After computing normalized phase variance, a variance threshold parameter $T_{n}$ is initialized to zero and is linearly increased until the cumulative range of frequencies for which phase variance is lower than $T_{n}$ achieves a fixed minimum frequency range $F_{\min }$ or $T_{n}$ hits a predetermined maximum value $T_{\max }$. This operation is performed independently on each available EEG channel. The Fourier components selected by the algorithm are restricted to lie in the passband of the bandpass filter used for preprocessing.

The signal is then reconstructed by taking the Inverse Fast Fourier Transform (IFFT). This type of filtering is adaptive to the signal since the signal itself determines the characteristics of the filter (defined in the Fourier domain). After the trial phase of the algorithm, during which the optimal filter is determined, all subsequent light averages are filtered in the same fashion.

\section{ADAPTIVE FILTERING IN THE SPACE-FREQUENCY DOMAIN USING A COMPLEX WAVELET TRANSFORM}

The standard discrete wavelet transform (DWT) has been shown to be useful for a wide range of signal processing applications (compression, digital image and video denoising). While the Fourier Transform is known to produce a uniform tiling of the time-frequency plane - with Fourier components that are welllocalized in frequency but not in time, wavelet coefficients provide a trade-off in time-frequency localization; highfrequency wavelets are well localized in time while lowfrequency ones are well localized in frequency. Dyadic wavelet analysis corresponds to tiling the time-frequency plane with "octave" frequency bands. In the one-dimensional case, the DWT is implemented by a filter bank made of bandpass filters whose passbands are $\left[f_{N} / 2, f_{N}\right],\left[f_{N} / 4, f_{N} / 2\right],\left[f_{N} / 8, f_{N} / 4\right]$, etc. where $f_{N}$ indicates the Nyquist frequency, i.e. one half of the sampling frequency. Wavelet transforms have been successfully used for denoising as long as the SNR is moderate to high, i.e. above $10 \mathrm{~dB}[5,6]$. When the signal is buried in high energy noise, i.e. with SNR of less than $0 \mathrm{~dB}$, it has been shown by Causevic et al. [2] that conventional wavelet denoising fails.

In addition, a drawback of the classical DWT is that it is not shift-invariant ${ }^{2}$ [13]. This results in the fact that the energy distribution between wavelet subbands is sensitive to a small time shift of the input signal. While this is of little importance for signal compression applications, it has been surmised that this lack of shift invariance might be the reason why wavelets have not been used very much for signal analysis [12]. The Complex Wavelet Transform (CWT), that was recently proposed

\footnotetext{
${ }^{2}$ The undecimated form of the dyadic wavelet decomposition tree (also referred to as "algorithme à trous") does not have this drawback but its computational complexity and high redundancy make it unattractive for many signal processing applications.
} 
by Kingsbury $[11,12]$ and introduces redundancy in the system, overcomes this deficiency of the classical DWT. It has been successfully used for image denoising as described for example in $[14,15]$. Perhaps more importantly, unlike real wavelet transforms, this complex transform preserves the notion of phase (and amplitude) of the transform coefficients, thereby making it possible to extend the algorithm described in Section 3.

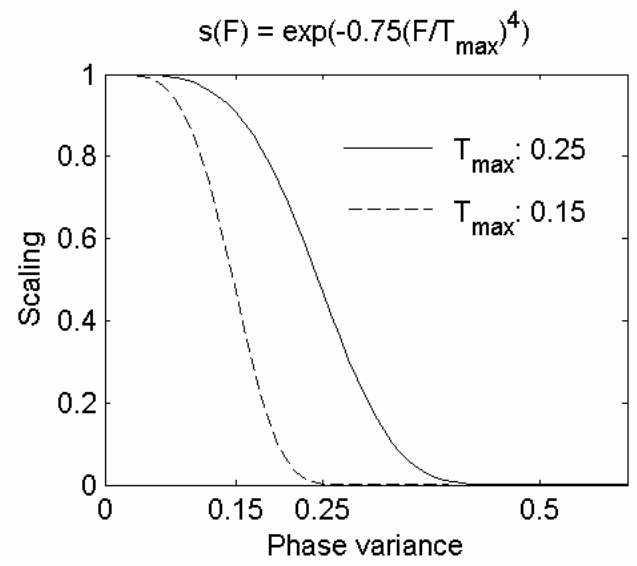

Figure 2: Behavior of scaling parameter as a function of normalized phase variance for two values of $T_{\max }$.

Our algorithm uses the Dual-Tree Complex Wavelet Transform (DCWT) of Selesnick [16] as the invertible transform in the adaptive filtering method of Fridman et al. The filters for this transform were first published by Kingsbury in [12]. In this case, the complex transform coefficients have a magnitude and a phase as is the case with the FFT. However, since wavelet coefficients are well localized in the time-frequency plane, setting the amplitude of a wavelet coefficient to zero will only affect a localized region in the time domain, whereas the equivalent operation in the FFT domain affects the signal over the entire frame. The transform size denoted by L was chosen to be 512 with 8 decomposition levels (scales) so that the lowresolution subband consisted of 2 coefficients.

After the CWT of each sub-average ("trial") is computed, the phase variance of each normalized wavelet coefficient $w_{i, j}$ is computed as it is in [8]. The magnitude of each wavelet coefficient is selectively scaled according to the phase variance of the coefficients at this location across the trials. In our implementation, this scaling has the form:

$$
w_{i, j}=\alpha_{i, j} \cdot W_{i, j} \exp \left(j \theta_{i, j}\right)
$$

where $W_{i, j}$ and $\theta_{i, j}$ are respectively the magnitude and phase of the unprocessed complex $i^{\text {th }}$ wavelet coefficient at the $j^{\text {th }}$ scale, and where:

$$
\alpha_{i, j}=\exp \left(-0.75 .\left(F_{i j} / T_{\max }\right)^{4}\right) \text {, }
$$

where $F_{i j}$ is the phase variance of coefficient $w_{i, j}$ across the trials. The behavior of this scaling parameter as a function of normalized phase variance is shown in Fig. 2. We found that this scaling was preferable to a "hard threshold", where it is set to zero for anything above $T_{\max }$. The parameter $T_{\max }$ should be a decreasing function of the length of the short-term average used since we expect longer averages to have a higher SNR and therefore have more coherence across trials.

The performance of the algorithm for the denoising of auditory brainstem evoked potentials is illustrated in the next section.

\section{EXPERIMENTAL RESULTS}

We denote by "BP + AVG" the method consisting of bandpass filtering followed by linear averaging, by "AFF" the method consisting of Adaptive Filtering in the Fourier domain and by "AFW" our new method consisting of Adaptive Filtering in the (complex) Wavelet domain. The parameters used in methods AFF and AFW with averages of length 512, 750 and 1024 are listed in Table I.

Average SNR results obtained for the three algorithms are given in Tables II(A-B) and are illustrated in Fig. 3.

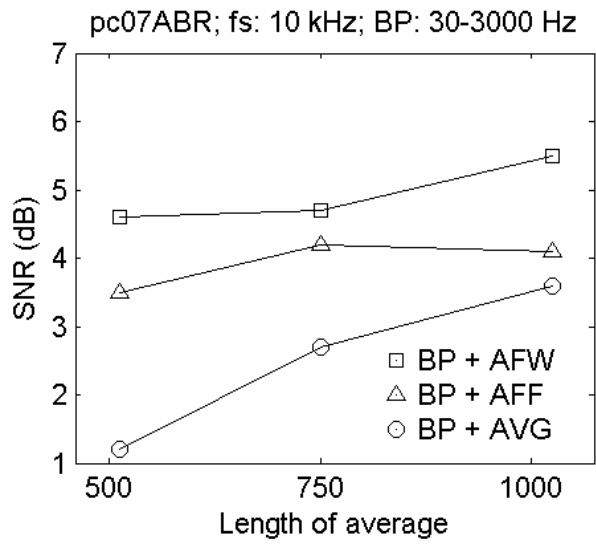

Figure 3: Comparative results of extracted signal quality (in $\mathrm{dB}$ ) as a function of average length.

Note by comparing the entries in Tables II(A) and II(B) the large absolute differences in SNR, for any same given method and parameters, between signals "pa04ABR" and "pa07ABR." This is because the average input SNR for the former signal is $3.4 \mathrm{~dB}$ higher than for the latter $(-23.2 \mathrm{~dB}$ vs. $-26.6 \mathrm{~dB})$. Furthermore, we see that the $\mathrm{BP}+\mathrm{AFW}$ algorithm significantly outperforms linear averaging in terms of SNR. Average SNR for this method is also consistently higher than for than for the FFT-based algorithm (BP + AFF).

\section{CONCLUSION}

In this work, we extended an algorithm for the extraction (denoising) of brainstem evoked potentials responses (ABR) by using a nearly shift-invariant Complex Wavelet Transform which scales wavelet coefficients based on the phase variance across successive subaverages. The performance of the novel algorithm was illustrated on two simulated EEG + EP signals where the underlying ideal EP signal is known, allowing objective comparisons of performance across algorithms. The wavelet-based algorithm outperforms the traditional bandpass filtering followed by averaging as well as a previously published FFT-based adaptive filtering algorithm. 
Table I: Algorithm parameters for AFF and AFW methods (Top-row: Length of "light average").

\begin{tabular}{|l|l|l|l|}
\hline & $\mathbf{5 1 2}$ & $\mathbf{7 5 0}$ & $\mathbf{1 0 2 4}$ \\
\hline AFF & $\mathrm{K}=8$ & $\mathrm{~K}=8$ & $\mathrm{~K}=8$ \\
& $\mathrm{~F}_{\text {min }}=950 \mathrm{~Hz}$ & $\mathrm{~F}_{\min }=950 \mathrm{~Hz}$ & $\mathrm{~F}_{\text {min }}=950 \mathrm{~Hz}$ \\
& $\mathrm{~T}_{\text {max }}=0.65$ & $\mathrm{~T}_{\max }=0.65$ & $\mathrm{~T}_{\text {max }}=0.65$ \\
& $\mathrm{P}=256$ & $\mathrm{P}=494$ & $\mathrm{P}=768$ \\
& $\mathrm{~L}=512$ & $\mathrm{~L}=512$ & $\mathrm{~L}=512$ \\
\hline AFW & $\mathrm{K}=8$ & $\mathrm{~K}=8$ & $\mathrm{~K}=8$ \\
& $\mathrm{~T}_{\text {max }}=0.25$ & $\mathrm{~T}_{\text {max }}=0.25$ & $\mathrm{~T}_{\text {max }}=0.15$ \\
& $\mathrm{P}=256$ & $\mathrm{P}=494$ & $\mathrm{P}=768$ \\
& $\mathrm{~L}=512$ & $\mathrm{~L}=512$ & $\mathrm{~L}=512$ \\
\hline
\end{tabular}

Table II: Comparison of SNR results for 3 EP extraction algorithms; SNR values in $\mathrm{dB}$ are given as: average (standard deviation) of a collection of SNR values over a time span of 1 minute (4000 epochs). (A) Signal: "pc04ABR" (input SNR: $23.2 \mathrm{~dB}$; sampling: $10 \mathrm{kHz}$ ); bandpass filter: $30-3000 \mathrm{~Hz}$; (B) Signal "pc07ABR" (input SNR: -26.6dB).

\begin{tabular}{|l|l|l|l|}
\hline pc04ABR & $\mathbf{5 1 2}$ & $\mathbf{7 5 0}$ & $\mathbf{1 0 2 4}$ \\
\hline BP + AVG & $6.5(3.2)$ & $7.8(3.6)$ & $8.8(3.9)$ \\
\hline BP + AFF & $7.4(3.5)$ & $8.3(3.7)$ & $9.2(3.9)$ \\
\hline BP + AFW & $8.4(3.2)$ & $9.1(3.4)$ & $10.1(3.8)$ \\
\hline
\end{tabular}

(A)

\begin{tabular}{|l|l|l|l|}
\hline pc07ABR & $\mathbf{5 1 2}$ & $\mathbf{7 5 0}$ & $\mathbf{1 0 2 4}$ \\
\hline BP + AVG & $1.2(3.1)$ & $2.7(3.4)$ & $3.6(3.5)$ \\
\hline BP + AFF & $3.5(2.9)$ & $4.2(3.0)$ & $4.1(3.0)$ \\
\hline BP + AFW & $4.6(2.8)$ & $4.7(2.8)$ & $5.5(2.9)$ \\
\hline
\end{tabular}

(B)

\section{REFERENCES}

[1] Bertrand, O., Bohorquez, J., and Pernier, J. Fast TimeFrequency Digital Filtering Based on an Invertible Wavelet Transform: An Application to Evoked Potentials. IEEE Trans. On Biomedical Engineering, Vol. 41, No. 1, January 1994.

[2] Causevic, E., Morley, R.E., Wickerhauser, M.V. and Jacquin, A. Fast Wavelet Estimation of Weak Biosignals. IEEE Trans. On Biomedical Engineering. To appear.

[3] Don, M., and Elberling, C. Use of quantitative measures of auditory brain-stem response peak amplitude and residual background noise in the decision to stop averaging. Journal of the Acoustical Society of America 99: 491-499, 1996.

[4] Don, M., Elberling, C. and Waring, M. Objective detection of averaged auditory brainstem responses. Scandinavian Audiology 13: 219-228, 1984.

[5] Donoho, D.L. De-noising by soft thresholding. IEEE Transactions on Information Theory. Vol. 41: 613-627, 1995.

[6] Donoho, D.L. and Johnstone, I. Ideal spatial adaptation via wavelet shrinkage. Biometrika, December, 81:425-455, 1994.
[7] Elberling, C., and Don, M. Quality estimation of averaged auditory brainstem responses. Scandinavian Audiology 13: 187197, 1984.

[8] Fridman, J., John, E.R., Bergelson, M., Kaiser, J.B., and Baird, H.W., Application of Digital Filtering and Automatic Peak Detection to Brainstem Auditory Evoked Potential, Electroencephalogy and Clinical Neurophysiology, 1982.

[9] Hall, J.W.III Handbook of Auditory Evoked Responses. Boston, Allyn and Bacon, 1992.

[10] Jewett, D.L. and Williston, J.S. Auditory evoked far fields averaged from the scalp of humans. Brain, 4, 681-696, 1971.

[11] Kingsbury, N.G., Complex wavelets for shift-invariant analysis and filtering of signals, Applied and Computational Harmonic analysis, 10(3):234-253, May 2001.

[12] Kingsbury, N.G., Image Processing with Complex Wavelets, Phil. Trans. R. Soc. London A, September 1999.

[13] Oppenheim A., Schafer, R. W., Digital Signal Processing, Prentice Hall, 1975.

[14] Sendur, L., and Selesnick, I.W., Bivariate shrinkage functions for wavelet-based denoising exploiting interscale dependency, IEEE Trans. On Signal Processing, 50(11): 2744$2756,2002$.

[15] Selesnick, I.W., A new complex-directional wavelet transform and its application to image denoising, IEEE International Conference on Image Processing (ICIP), 2002.

[16] Selesnick, I. http://taco.poly.edu/WaveletSoftware/dt1D.html

[17] Spehlmann, Misulis, K.E., Fakhoury, T., Evoked Potential Primer, Elsevier Science \& Technology Books, 2001. 